\title{
The quantum free-electron laser
}

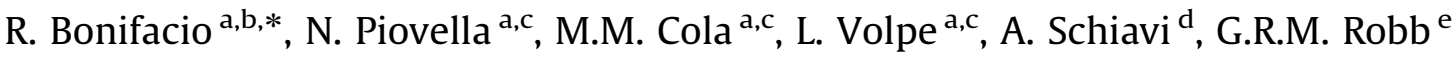 \\ a INFN, Sezione di Milano, via Celoria 16, I-20133 Milano, Italy \\ ${ }^{\mathrm{b}}$ Centro Brasileiro de Pesquisas Físicas, Rio de Janeiro, Brazil \\ c Dipartimento di Fisica, Università Degli Studi di Milano, via Celoria 16, I-20133 Milano, Italy \\ 'Dipartimento di Energetica, Università di Roma "La Sapienza" and INFN, Via Scarpa 14, I-00161 Roma, Italy \\ e SUPA, Department of Physics, University of Strathclyde, Glasgow G4 0NG, Scotland, UK
}

\section{A R T I C L E I N F O}

Available online 6 May 2008

Keywords:

SASE

$\mathrm{X}$-ray laser

Line width

\begin{abstract}
A B S T R A C T
A Free-Electron Laser (FEL) operating in the quantum regime can provide a compact and monochromatic X-ray source. Here we review the basic principles of a high-gain quantum FEL starting from noise, with special emphasis on the self-amplified spontaneous emission (SASE) mode operation. In the first part, the full quantum theory of the $N$-particle and single-radiation-mode FEL Hamiltonian is presented. Quantum effects such as cooperative gain, discrete spectrum and line narrowing are described, both in the multi-particle and in the second quantization formalism. In the second part, propagation effects (i.e. slippage) are described and the main features of the quantum SASE regime are discussed. The broad and spiky radiation spectrum observed in the classical SASE reduces in the quantum regime to a series of narrow lines, associated to sequential transitions between adjacent momentum states. A simple interpretation of the discrete nature of the spectrum and of the line width of the single spike observed in the quantum regime is presented.
\end{abstract}

(c) 2008 Elsevier B.V. All rights reserved.

\section{Classical and quantum SASE-FEL}

The realization of a Free-Electron Laser (FEL) in the selfamplified spontaneous emission (SASE) mode [1] is presently the goal of several projects (such as LCLS [2] at Stanford, USA, XFEL [3] in Hamburg, Germany, and [4] in Japan), to obtain a highbrightness X-ray source. However, such sources will radiate a pulse with a broad spectrum composed of many random superradiant spikes [5]. Recently it has been shown that an FEL can operate in a quantum regime [6], in which the spiking behavior observed in SASE mode disappears and the spectrum reduces to a single narrow line $[7,8]$, providing an enormous improvement in the coherence of SASE-FEL based X-ray sources. The transition from the classical to the quantum regime is controlled by the "quantum FEL (QFEL) parameter"

$\bar{\rho}=\rho \frac{m c \gamma_{\mathrm{r}}}{\hbar k}$

(where $\rho$ is the FEL parameter [1]), equal to the ratio between the maximum classical momentum spread (of the order of $m c \gamma_{\mathrm{r}} \rho$ ) and the photon recoil momentum $\hbar k$. It expresses also the maximum number of photons emitted per electron in the classical regime. When $\bar{\rho} \gg 1$ an SASE-FEL operates in the classical "multi-photon"

\footnotetext{
* Corresponding author at: INFN, Sezione di Milano, via Celoria 16, I-20133 Milano, Italy.

E-mail address: rodolfomcz@gmail.com (R. Bonifacio).
}

regime, and the spectrum of the emitted field is broad and chaotic. In this regime an SASE-FEL radiates a random series of superradiant spikes because, at short wavelength, the electron bunch contains many cooperation lengths which radiate randomly and independently one from the other [5]. The number of spikes in the high-gain regime corresponds approximately to the number of cooperation lengths in the electron bunch (i.e. $L_{\mathrm{b}} / 2 \pi L_{\mathrm{c}}$ ). The final result is an almost chaotic temporal pulse structure with a broad spectral width, unless $L_{\mathrm{b}} \leqslant 2 \pi L_{\mathrm{c}}$. Hence, classical SASE has the following drawback with regard to its application as a useful source of short-wavelength coherent light: when $L_{\mathrm{b}} \gg 2 \pi L_{\mathrm{c}}$, its temporal coherence is very poor due to the noisy spectrum.

Recently, we have shown that when an SASE-FEL is in the quantum regime (with $\bar{\rho} \leqslant 1$ ) each electron emits only a single photon and the spectrum reduces to a single narrow line, Fourier limited by the electron beam duration [9]. So the spiky spectrum of classical SASE is replaced by an almost coherent spectrum, as if the system would be driven by a coherent seed. More specifically, in the quantum regime the spectrum is composed by discrete narrow lines equally spaced, generated by sequential transitions between adjacent momentum states. Increasing $\bar{\rho}$, the line distance decreases and the width increases until when, for $\bar{\rho}>0.4$, the lines overlap and the continuous and spiky classical spectrum is recovered.

For an experimental realization of a QFEL it is necessary to use a laser wiggler in a Compton backscattered configuration [10,11], 
instead of the magnetic wiggler as used in the classical SASE experiments [2-4]. In a laser wiggler configuration, a low-energy electron beam back scatters the photons of a counter-propagating high power laser, with a frequency up-shifted by a factor $4 \gamma^{2}$. However, such a choice sets some stringent conditions on the electron and laser beam parameters. A parametric study of the experimental requirements necessary to operate a laser wiggler FEL in the quantum regime has been discussed in Ref. [9].

\section{Quantum description of FEL}

We review the main features of the quantum description of an FEL, limited to the one-dimensional, cold electron beam and uniform wiggler case. The more general three-dimensional quantum model of an FEL with the laser wiggler is described elsewhere in these Proceedings. Following Ref. [8], we start from the FEL Hamiltonian for $N$ electrons interacting with a single mode of radiation $[12,13]$ :

$H=\sum_{j=1}^{N}\left[\frac{p_{j}^{2}}{2 \bar{\rho}}+\mathrm{i} \sqrt{\bar{\rho} / N}\left(a^{\dagger} \mathrm{e}^{-\mathrm{i} \theta_{j}}-a \mathrm{e}^{\mathrm{i} \theta_{j}}\right)\right]-\frac{\delta}{N} a^{\dagger} a$

where $\theta_{j}=\left(k+k_{\mathrm{w}}\right) z-c k t_{j}-\delta \bar{z}$ and $p_{j}=m c\left(\gamma_{j}-\gamma_{0}\right) / \hbar\left(k+k_{\mathrm{w}}\right)$ are position and momentum operators of the $j$-th electron, with $\left[\theta_{i}, p_{j}\right]=\mathrm{i} \delta_{i j}, a$ is the annihilation operator of the radiation field, with $\left[a, a^{\dagger}\right]=1, \bar{z}=z / L_{\mathrm{g}}, L_{\mathrm{g}}=\lambda_{\mathrm{w}} / 4 \pi \rho$ is the gain length, $\delta=$ $\left(\gamma_{0}-\gamma_{\mathrm{r}}\right) / \rho \gamma_{0}$ is the detuning, $\rho=\left(1 / \gamma_{\mathrm{r}}\right)\left(a_{\mathrm{w}} \omega_{\mathrm{p}} / 4 c k_{\mathrm{w}}\right)^{2 / 3}$ is the classical FEL parameter, $\omega_{\mathrm{p}}=\sqrt{e^{2} n / m \varepsilon_{0}}$ is the plasma frequency, $\gamma_{\mathrm{r}}=\sqrt{k\left(1+a_{\mathrm{w}}^{2}\right) / 2 k_{\mathrm{w}}}$ is the resonant energy (in $m c^{2}$ units) and $a_{\mathrm{w}}$ is the undulator parameter. We observe that the dynamics depend on the QFEL parameter $\bar{\rho}$ defined in Eq. (1). From the Hamiltonian (2) we derive the following Heisenberg equations for the operators:

$$
\begin{aligned}
& \frac{\mathrm{d} \theta_{j}}{\mathrm{~d} \bar{z}}=\frac{p_{j}}{\bar{\rho}} \\
& \frac{\mathrm{d} p_{j}}{\mathrm{~d} \bar{z}}=-\sqrt{\bar{\rho} / N}\left(a \mathrm{e}^{\mathrm{i} \theta_{j}}+a^{\dagger} \mathrm{e}^{-\mathrm{i} \theta_{j}}\right) \\
& \frac{\mathrm{d} a}{\mathrm{~d} \bar{z}}=\sqrt{\bar{\rho} / N} \sum_{j=1}^{N} \mathrm{e}^{-\mathrm{i} \theta_{j}}+\mathrm{i} \delta a .
\end{aligned}
$$

A constant of motion, which represents the total momentum in dimensionless units, is given by

$\sum_{j=1}^{N} p_{j}+a^{\dagger} a=$ const.

Let us introduce the following electron collective operators:

$B=\frac{1}{\sqrt{N}} \sum_{j=1}^{N} \mathrm{e}^{-\mathrm{i} \theta_{j}}$

$P=\frac{1}{\sqrt{N}} \sum_{j=1}^{N}\left(\frac{p_{j} \mathrm{e}^{-\mathrm{i} \theta_{j}}+\mathrm{e}^{-\mathrm{i} \theta_{j}} p_{j}}{2}\right)$

where $B$ is the bunching and $P$ is the symmetrized momentum bunching. This symmetrization is fundamental whenever one is dealing with products of non-commuting operators, i.e. $\left[\mathrm{e}^{-\mathrm{i} \theta_{j}}, p_{k}\right]=\delta_{j k} \mathrm{e}^{-\mathrm{i} \theta_{j}}$.

We consider $a, p_{j}$ and $\sum_{j} \mathrm{e}^{-\mathrm{i} \theta_{j}}$ as fluctuation operators, i.e. the initial states for the electrons and the field is such that $\langle a\rangle_{0}=\left\langle p_{j}\right\rangle_{0}=\sum_{j}\left\langle\mathrm{e}^{-\mathrm{i} \theta_{j}}\right\rangle_{0}=0$. Writing the Heisenberg equations of motion and neglecting the high-order quantities

$\frac{1}{\sqrt{N}} \sum_{j}\left(p_{j} \mathrm{e}^{-\mathrm{i} \theta_{j}} p_{j}\right), \quad a^{\dagger} \frac{1}{N} \sum_{j} \mathrm{e}^{-2 \mathrm{i} \theta_{j}}$ we obtain the following equations for the linear regime:

$$
\begin{aligned}
& \frac{\mathrm{d} B}{\mathrm{~d} \bar{z}}=-\frac{\mathrm{i}}{\bar{\rho}} P \\
& \frac{\mathrm{d} P}{\mathrm{~d} \bar{z}}=-\frac{\mathrm{i}}{4 \bar{\rho}} B-\sqrt{\bar{\rho}} a \\
& \frac{\mathrm{d} a}{\mathrm{~d} \bar{z}}=\sqrt{\bar{\rho}} B+\mathrm{i} \delta a .
\end{aligned}
$$

The quantum correction to the classical description [1] is given by the term $-\mathrm{i} B / 4 \bar{\rho}$ in the equation for $P$. Looking for solutions of the linear system (10)-(12) of the form $B(\bar{z})=B_{0} \exp (\mathrm{i} \lambda \bar{z})$, we obtain the cubic characteristic equation

$(\lambda-\delta)\left(\lambda^{2}-\frac{1}{4 \bar{\rho}^{2}}\right)+1=0$.

Notice that this dispersion relation coincides with that of a classical FEL with an initial energy spread with a square distribution and width $1 / 2 \bar{\rho}$, i.e. this extra term represents the intrinsic quantum momentum spread which, in real units, becomes $\hbar k / 2$. The features of the solution of the cubic equation (13) are shown in Fig. 1 . When $\bar{\rho} \leqslant 1$ (Fig. 1(b)-(f)), the resonance occurs at $\delta=1 /(2 \bar{\rho})$, with full width equal to $4 \sqrt{\bar{\rho}}$ and peak value $\operatorname{Im} \lambda=\sqrt{\bar{\rho}}$. Note that the field and the bunching grow exponentially as $\exp (\sqrt{\bar{\rho}} \bar{z})=\exp \left(z / L_{\mathrm{g}}^{\prime}\right)$, where $L_{\mathrm{g}}^{\prime}=L_{\mathrm{g}} / \sqrt{\bar{\rho}}=\lambda_{\mathrm{w}} /(4 \pi \rho \sqrt{\bar{\rho}})$ is the quantum gain length.

Hence, in the quantum regime $\bar{\rho}<1$, the resonance condition is $m c\left(\gamma_{\mathrm{r}}-\gamma_{0}\right)=\hbar k / 2$ (i.e. $\delta=1 / 2 \bar{\rho}$ our dimensionless variables). Moreover, the gain bandwidth increases (and the gain length decreases) as the square root of $\bar{\rho}$.

An alternative description to the $N$-particle Hamiltonian model can be formulated in the second-quantization formalism, treating the electrons as non-interacting bosons [14,15]. In this formulation, the $N$ particles are described by a matter-field operator $\hat{\Psi}(\theta, \bar{z})$ obeying the bosonic equal-time commutation relation

$\left[\hat{\Psi}(\theta), \hat{\Psi}(\theta)^{\dagger}\right]=\delta\left(\theta-\theta^{\prime}\right)$

and the normalization condition is

$\int_{0}^{2 \pi} \hat{\Psi}(\theta)^{\dagger} \hat{\Psi}(\theta)=\hat{N}$

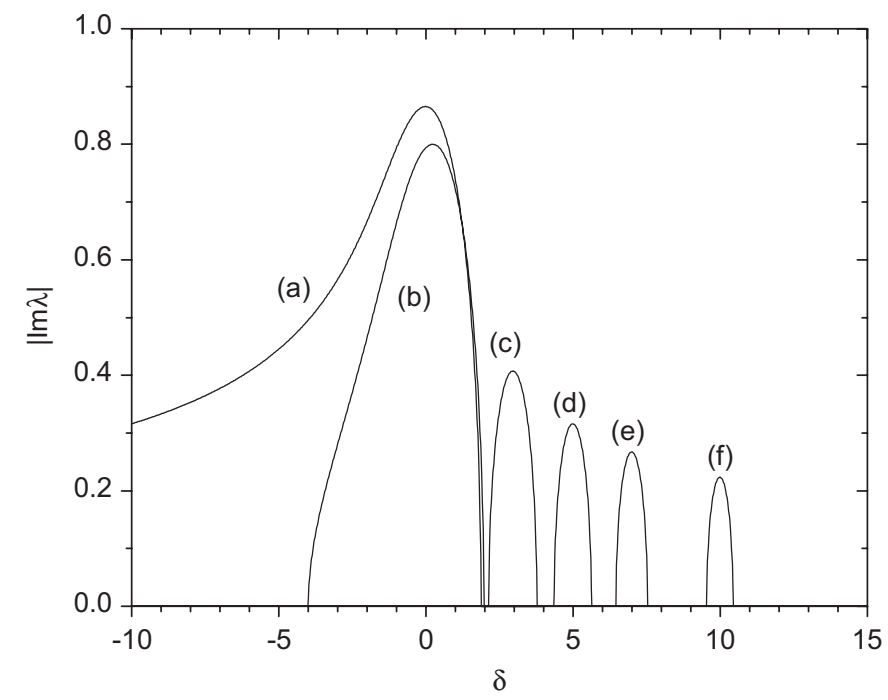

Fig. 1. Imaginary part of the complex root of the cubic Eq. (13) vs. $\delta$ for $1 / 2 \bar{\rho}$ equal to (a) 0 , (b) 0.5 , (c) 3, (d) 5, (e) 7 and (f) 10 . 
In this formulation, the second-quantized Hamiltonian is

$\hat{H}=\int_{0}^{2 \pi} \hat{\Psi}(\theta)^{\dagger} H\left(\theta,-\mathrm{i} \frac{\partial}{\partial \theta}, a, a^{\dagger}\right) \hat{\Psi}(\theta)$

where $H$ is the single-particle Hamiltonian defined in (2). The Heisenberg equation for $\hat{\Psi}(\theta, \bar{z})$ and $a$ are

$\mathrm{i} \frac{\partial \hat{\Psi}}{\partial \bar{z}}=[\hat{\Psi}, \hat{H}]=-\frac{1}{2 \bar{\rho}} \frac{\partial^{2} \hat{\Psi}}{\partial \theta^{2}}+\mathrm{i} \sqrt{\bar{\rho} / N}\left(a^{\dagger} \mathrm{e}^{-\mathrm{i} \theta}-a \mathrm{e}^{\mathrm{i} \theta}\right) \hat{\Psi}$

$\frac{\mathrm{d} a}{\mathrm{~d} \bar{z}}=-\mathrm{i}[a, \hat{H}]=\sqrt{\bar{\rho} / N} \int_{0}^{2 \pi} \mathrm{d} \theta \hat{\Psi}(\theta)^{\dagger} \mathrm{e}^{-\mathrm{i} \theta} \hat{\Psi}(\theta)+\mathrm{i} \delta a$.

We expand the matter-wave field in the momentum basis,

$\hat{\Psi}(\theta)=\sum_{n=-\infty}^{+\infty} c_{n} u_{n}(\theta)$

where $u_{n}=(1 / \sqrt{2 \pi}) \exp (\mathrm{im} \theta)$ are the eigenfunctions of $p$ with eigenvalue $n$ and $c_{n}$ are the annihilation operators for the state with eigenvalue $n$, with $\left[c_{n}, c_{m}^{\dagger}\right]=\delta_{n, m}$. Then, using (19) in Eqs. (17) and (18), we obtain

$$
\begin{aligned}
& \frac{\mathrm{d} c_{n}}{\mathrm{~d} \bar{z}}=-\mathrm{i} \frac{n^{2}}{2 \bar{\rho}} c_{n}+\sqrt{\bar{\rho} / N}\left(a^{\dagger} c_{n+1}-a c_{n-1}\right) \\
& \frac{\mathrm{d} a}{\mathrm{~d} \bar{z}}=\sqrt{\bar{\rho} / N} \sum_{n=-\infty}^{+\infty} c_{n-1}^{\dagger} c_{n}+\mathrm{i} \delta a .
\end{aligned}
$$

The quantum expression for the bunching parameter appearing in the right-hand side of Eq. (21)

$b=\frac{1}{N} \sum_{n=-\infty}^{+\infty} c_{n-1}^{\dagger} c_{n}$

shows that the electron bunching involves a coherent superposition of different momentum states. A fully quantum treatment of the linear regime of Eqs. (20) and (21) has been given in Ref. [15], considering the equilibrium state with no photons and all the electrons in the state with $n=0$ (i.e. $\langle a\rangle_{0}=0$ and $\left.\left\langle c_{0}^{\dagger} c_{0}\right\rangle_{0}=N\right)$. Then, considering $c_{1}, c_{-1}$ and $a$ as fluctuation operators, we obtain the same quantum linear equations (10)-(12), in which the bunching and the momentum bunching operators are defined as $B=c_{1}+c_{-1}^{\dagger}$ and $P=c_{1}-c_{-1}^{\dagger}$. In this description the electrons have initially a definite value of momentum (i.e. $p=0$ ), so that they are delocalized in position. The dynamics of the system is that of three parametric coupled harmonic oscillators, $a_{1}=c_{-1}, a_{2}=c_{1}$ and $a_{3}=a$, which obey commutation rules $\left[a_{i}, a_{j}\right]=0$ and $\left[a_{i}, a_{j}^{\dagger}\right]=\delta_{i j}$ for $i, j=1,2,3$. The average occupation numbers are $\left\langle n_{i}\right\rangle=\left\langle a_{i}^{\dagger} a_{i}\right\rangle(i=1,2,3)$. The state $n_{1}$ refers to electrons with negative recoil (decelerating), $n_{2}$ with positive recoil (accelerating) and $n_{3}$ is the photon number. In Ref. [15] the exact evolution of the three modes has been calculated starting from vacuum fluctuations, demonstrating that the three modes are entangled and the number variance is $\sigma_{i}^{2}=$ $\left\langle n_{i}\right\rangle\left(1+\left\langle n_{i}\right\rangle\right)[12,13]$, i.e. the statistics is that of a thermal state. Furthermore, in Ref. [15] it has been shown that for $\bar{\rho} \leqslant 1$ the electrons, initially in the momentum state $n=0$, populate only the lower momentum state $n=-1$, recoiling backward by $\hbar k$ when a photon is emitted. In this quantum regime the system behaves as a two-level system, described by the two operators $c_{0}$ and $c_{-1}$. The average number of photons grows exponentially as $\left\langle n_{3}\right\rangle \approx\left\langle n_{1}\right\rangle \approx\left(\frac{1}{4}\right) \exp (\sqrt{\bar{\rho}} \bar{z})$ at resonance (i.e. for $\left.\delta=1 /(2 \bar{\rho})\right)$ and at saturation the number of emitted photons is $N$.

\section{QFEL model with propagation}

The previous quantum model has been extended to include the effects of propagation or slippage [16] (which are fundamental to
SASE) by using a multiple scaling method, previously adopted also to derive the classical FEL equations [17]. This allows to take into account the existence of two different spatial length scales: the variation of the electron distribution on the scale of the radiation wavelength (describing the bunching on the variable $\theta$ ) and the variation of the field envelope on the much longer scale of the cooperation length, described by $z_{1}=2 \rho \theta=\left(z-v_{\mathrm{r}} t\right) / \beta_{\mathrm{r}} L_{\mathrm{c}}$, i.e. the electron coordinate along the bunch, in units of the cooperation length, $L_{\mathrm{c}}=\lambda /(4 \pi \rho)$ [18]. As discussed in Ref. [14], the field operator $\hat{\Psi}$ can be approximated by the complex function $\Psi \approx\langle\hat{\Psi}\rangle / \sqrt{N}$ and the electromagnetic field by the dimensionless classical radiation amplitude, $A=a / \sqrt{\bar{\rho} N}$. With these approximations, the QFEL model with propagation is

$$
\begin{gathered}
\begin{aligned}
& \mathrm{i} \frac{\partial \Psi\left(\theta, z_{1}, \bar{z}\right)}{\partial \bar{z}}=-\frac{1}{2 \bar{\rho}} \frac{\partial^{2}}{\partial \theta^{2}} \Psi\left(\theta, z_{1}, \bar{z}\right) \\
&-\mathrm{i} \bar{\rho}\left[A\left(z_{1}, \bar{z}\right) \mathrm{e}^{\mathrm{i} \theta}-\text { c.c. }\right] \Psi\left(\theta, z_{1}, \bar{z}\right)
\end{aligned} \\
\begin{aligned}
& \frac{\partial A\left(z_{1}, \bar{z}\right)}{\partial \bar{z}}+\frac{\partial A\left(z_{1}, \bar{z}\right)}{\partial z_{1}}= \int_{0}^{2 \pi} \mathrm{d} \theta\left|\Psi\left(\theta, z_{1}, \bar{z}\right)\right|^{2} \mathrm{e}^{-\mathrm{i} \theta} \\
&+\mathrm{i} \delta A\left(z_{1}, \bar{z}\right) .
\end{aligned}
\end{gathered}
$$

Notice that $A$ is defined such that $\bar{\rho}|A|^{2}$ is the ratio between the photon and the electron densities. Moreover, From Eq. (23) it follows that the dimensionless density profile

$I_{0}\left(z_{1}\right)=\int_{0}^{2 \pi}\left|\Psi\left(\theta, z_{1}, \bar{z}\right)\right|^{2} \mathrm{~d} \theta$

is independent of $\bar{z}$. This means that the spatial distribution of the particles does not change appreciably on the slow scale $z_{1}$ during the interaction with the radiation field.

Eqs. (23) and (24) are conveniently solved in the momentum representation. Assuming that $\Psi\left(\theta, z_{1}, \bar{z}\right)$ is a periodic function of $\theta$, it can be written as a Fourier series of momentum eigenstates as in Eq. (19):

$\Psi\left(\theta, z_{1}, \bar{z}\right)=\frac{1}{\sqrt{2 \pi}} \sum_{n=-\infty}^{\infty} c_{n}\left(z_{1}, \bar{z}\right) \mathrm{e}^{\mathrm{i} n(\theta+\delta \bar{z})}$

where now $\left|c_{n}\left(z_{1}, \bar{z}\right)\right|^{2}$ is the local probability to have an electron with momentum $p=n(\hbar k)$ at $\bar{z}$ and $z_{1}$. By inserting Eq. (26) into Eqs. (23) and (24) and defining $A=\bar{A} \mathrm{e}^{\mathrm{i} \delta \bar{z}}$, we obtain [16]

$\frac{\partial c_{n}}{\partial \bar{Z}}=-\mathrm{i} E_{n} c_{n}-\bar{\rho}\left(\bar{A} c_{n-1}-\bar{A}^{*} c_{n+1}\right)$

$\frac{\partial \bar{A}}{\partial \bar{z}}+\frac{\partial \bar{A}}{\partial z_{1}}=\sum_{n=-\infty}^{\infty} c_{n} c_{n-1}^{*}$

where $E_{n}=n^{2} /(2 \bar{\rho})+n \delta$. Eqs. (27) and (28) are the discrete QFEL model with propagation.

\subsection{Linear analysis}

We now perform a stability analysis of Eqs. (27) and (28) around the equilibrium state in which the electrons are in the momentum state $n(\hbar k)$, with arbitrary $n$. As done in Section 2, we assume $\bar{A}=0$ and $c_{n}=1$ and $c_{m}=0$ for all $m \neq n$, i.e. all the electrons in the same state $n$. Looking for solutions of the linearized equations proportional to $\mathrm{e}^{\mathrm{i}\left(\lambda \bar{z}+\bar{\omega} z_{1}\right)}$ (where $\bar{\omega}=$ $\left(L_{c} / c\right)\left(\omega^{\prime}-\omega\right)=\left(\omega^{\prime}-\omega\right) /(2 \rho \omega)$ is the frequency shift of the radiation field with respect to the carrier frequency $\omega$ ), one obtains the quantum dispersion relation

$\left(\lambda-\Delta_{n}\right)\left(\lambda^{2}-\frac{1}{4 \bar{\rho}^{2}}\right)+1=0$

where $\Delta_{n}=\delta+(n / \bar{\rho})-\bar{\omega}$ is the generalized detuning. Note that the dispersion relation in Eq. (29) reduces to Eq. (13) when $n=0$ and $\bar{\omega}=0$. The behavior of the imaginary part of $\lambda$ as a function of 
a

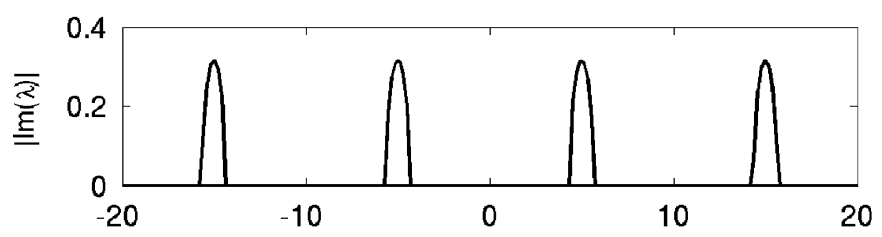

b

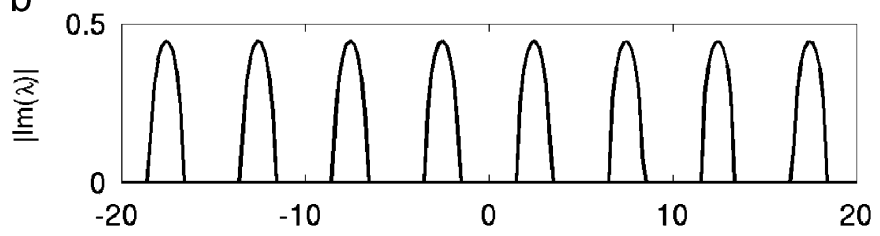

C

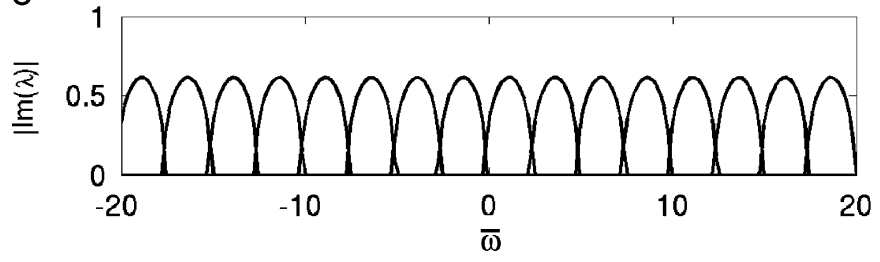

Fig. 2. Imaginary part of the unstable root of the cubic equation (29) vs. $\bar{\omega}=$ $\left(\omega^{\prime}-\omega\right) /(2 \rho \omega)$ for $\delta=0$, (a) $\bar{\rho}=0.1$, (b) $\bar{\rho}=0.2$, and (c) $\bar{\rho}=0.4$. Each line is centered around $\bar{\omega}=\left(n-\frac{1}{2}\right) / \bar{\rho}$ and has a width $4 \sqrt{\bar{\rho}}$. For $\bar{\rho}>0.4$ the lines overlap and the spectrum becomes almost continuous.

$\Delta_{n}$ is the same as that shown in Fig. 1 , in which $\Delta_{n}$ takes the place of $\delta$. We remember that, when $\bar{\rho}<1$, the resonance moves from $\Delta_{n}=0$ to $\Delta_{n}=1 /(2 \bar{\rho})$, with a width of $4 \sqrt{\bar{\rho}}$ in units of $\Delta_{n}$. This corresponds, in the momentum space, to a shift of $\hbar k / 2$ with a width $4 \bar{\rho}^{3 / 2}(\hbar k)$. Let us now consider a fixed value of $\bar{\rho}$ and plot $\operatorname{Im}(\lambda)$ for $\delta=0$ as a function of frequency shift $\bar{\omega}$, as shown in Fig. 2. It can be seen that the regions of the spectrum corresponding to gain $(\operatorname{Im}(\lambda)>0)$ appear as a series of discrete lines corresponding to different values of $n$. Each of these lines is centered on $\bar{\omega}=\left(n-\frac{1}{2}\right) / \bar{\rho}$, equally separated by a distance $1 / \bar{\rho}$, and has a width $4 \sqrt{\bar{\rho}}$. The transition to the classical continuous gain spectrum occurs when the width of the lines becomes larger than their separation, i.e. when $\bar{\rho}>\left(\frac{1}{2}\right)^{4 / 3} \approx 0.4$ (see Fig. 2). The physical reason for the existence of such a discrete spectrum is that in the quantum regime the electron recoils by $\hbar k$, so it undergoes a transition from an initial state with energy $E_{n} \propto p^{2} \propto n^{2}$, to the final state with energy $E_{n-1} \propto(n-1)^{2}$. Hence, the transition frequency varies as $n-\frac{1}{2}$, as shown above. For $\bar{\rho} \gg 1$ the probabilities of transition from the momentum states $n$ to $n \pm 1$ are comparable (i.e. $\left|c_{n+1}\right|^{2} \approx\left|c_{n-1}\right|^{2}$ ), so that an electron may either absorb or emit a photon. The difference between the emission and absorption rates yields the FEL gain in the classical regime. On the contrary, in the case $\bar{\rho}<1$, the emission rate is much larger than the absorption rate (i.e. $\left|c_{n-1}\right|^{2} \gg\left|c_{n+1}\right|^{2}$ ) and the electrons do only the transition $n \rightarrow n-1$, emitting a photon. In this quantum regime the FEL behaves approximately as a twolevel system described by the Maxwell-Bloch equations known in laser physics [19].

\subsection{The quantum purification of the SASE spectrum}

We now show that the discrete nature of the gain spectrum in the quantum regime, shown in Fig. 2, is the origin of the "quantum purification" observed the SASE spectrum. Fig. 3 shows the numerical solution of Eqs. (27) and (28), for $L_{\mathrm{b}}=30 L_{\mathrm{c}}$ and $\delta=0$. The simulations assume all the electrons initially in the same momentum state $n=0, A\left(z_{1}, \bar{z}=0\right)=0, \quad c_{-1}\left(z_{1}, \bar{z}=0\right)=$ $b_{0} \mathrm{e}^{\mathrm{i} \phi\left(z_{1}\right)}$ and $c_{0}\left(z_{1}, \bar{z}=0\right)=\sqrt{1-b_{0}^{2}}$, where $b_{0}=0.01$ and $\phi\left(z_{1}\right)$ is a randomly fluctuating phase with values in the range $[0,2 \pi)$. Fig. 3(a) and (b) show the field intensity as a function of $z_{1}$ at $\bar{z}=30$ for the classical regime and $\bar{z}=150$ for the quantum regime, respectively. Fig. 3(c) and (d) show the corresponding classical and quantum power spectra of the radiated field vs. $\bar{\omega}=\left(\omega^{\prime}-\omega\right) / 2 \rho \omega$, where $\omega$ is the carrier frequency. A dramatic difference between the classical (Fig. 3(a) and (c)) and the quantum evolution (Fig. 3(b) and (d)) appears: whereas the temporal structure in the classical limit is almost chaotic and spectrum broad (see Fig. 3(a) and (c)), on the contrary in the quantum limit the temporal behavior shows a purification of the initially noisy evolution, and the spectrum is composed of two narrow lines, whose positions are in agreement with the linear theory prediction (see Fig. 2(a)). Notice that the line separation $1 / \bar{\rho}$ corresponds in frequency to the relativistic recoil frequency $2 \hbar k^{2} / \gamma_{\mathrm{r}} m$. In Fig. 3(b) we observe also the rapid beat between the two frequencies of Fig. 3(d). Initially, for short $\bar{z}$, only the frequency with $\bar{\omega}=-1 /(2 \bar{\rho})$ appears. Increasing $\bar{z}$ additional lines downshifted by $1 / \bar{\rho}$ also appear, corresponding to the sequence of the several momentum transitions.

The transition from the quantum to the classical SASE regimes is observed in Fig. 4, showing the scaled power spectra, $P(\bar{\omega})$, for different values of $\bar{\rho}$ and for $\bar{z}=150$. We observe that the spectrum, composed of discrete, narrow lines for $\bar{\rho} \ll 1$, becomes quasi-continuous when $\bar{\rho} \geqslant 0.4$, in agreement with the predictions of the linear analysis described in the previous section.

\subsection{A simple interpretation of the spectral narrowing}

The "quantum purification" of the SASE spectrum can be interpreted by the following simple argument [9]: The maximum induced energy spread in an FEL is $\delta \gamma / \gamma \sim \rho$, which in terms of momentum spread is $\delta p=m c \delta \gamma \sim \bar{\rho}(\hbar k)$. So, the QFEL parameter $\bar{\rho}$ yields the ratio between the maximum momentum spread (induced in the classical regime) and the photon recoil momentum $\hbar k$; quantum effects become important when $\bar{\rho}<1$, since in this case the discreteness of momentum exchange is relevant. This provides a simply explanation of the origin of the broad and spiky classical spectrum and its reduction to a single line in the quantum regime. In fact, the radiation emission is due to the transition between adjacent recoil momentum states $\left(p^{(n)}=n \hbar k\right)$, which are equally spaced by the photon momentum. The emitted frequencies in the transitions $n \rightarrow n-1$ are also equally spaced, since they are proportional to the difference between the corresponding kinetic energies. In the classical regime $(\bar{\rho} \gg 1)$ many momentum states becomes occupied and the multiple transitions between the different momentum states lead to a multi-frequency spectrum with equally spaced lines and an envelope width $\Delta \omega / \omega \sim 2 \rho$. The many sequential transitions occur randomly under the gain curve, leading to the multiple-line chaotic spectrum observed in the classical SASE. Since the radiation is emitted during a time $L_{\mathrm{b}} / c$, its line width is $\Delta \omega / \omega \simeq \lambda / L_{\mathrm{b}}$. Hence, the number of spikes $N_{\mathrm{s}}$ in the classical regime can be obtained by the ratio between the envelope width and the single spike line width $N_{\mathrm{s}}=2 \rho /\left(\lambda / L_{\mathrm{b}}\right)=L_{\mathrm{b}} /\left(2 \pi L_{\mathrm{c}}\right)$ [5], where $L_{\mathrm{c}}=\lambda / 4 \pi \rho$ is the cooperation length. Conversely, in the quantum regime $\bar{\rho}<1$, the momentum spread $\delta p$ cannot be smaller than the photon recoil $\hbar k$ and only a single frequency, corresponding to a single momentum transition, occurs with line width

$$
\left(\frac{\Delta \omega}{\omega}\right)_{\mathrm{QFEL}} \simeq \frac{\lambda}{L_{\mathrm{b}}} .
$$


a

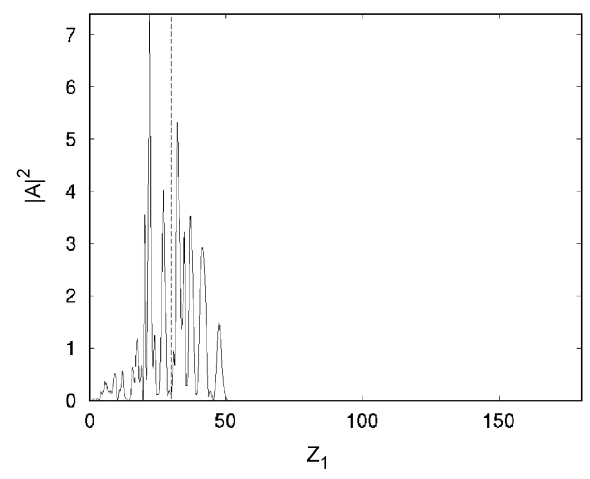

C

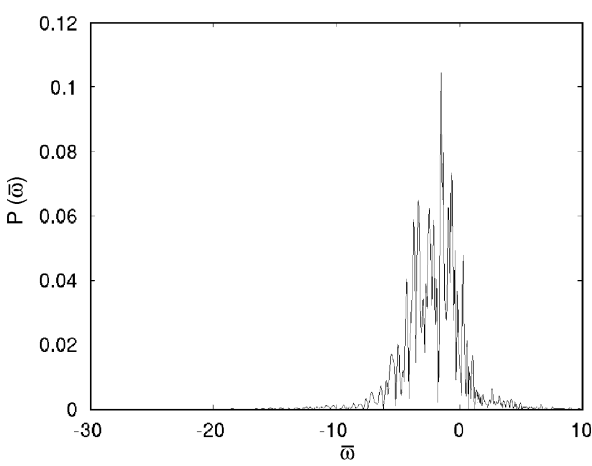

b

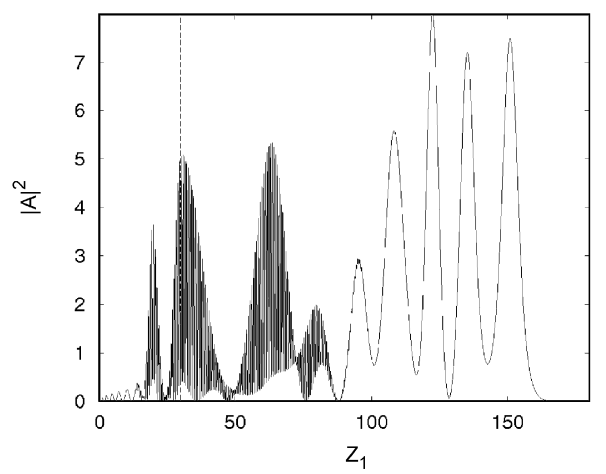

d

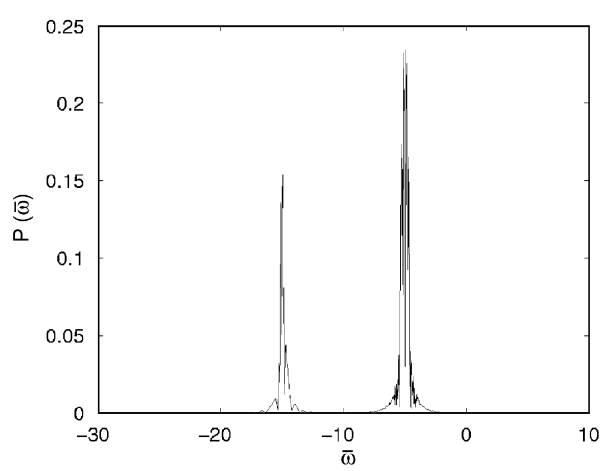

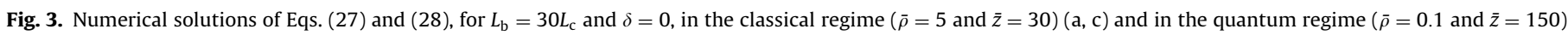

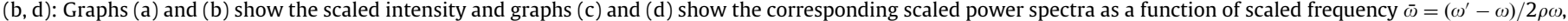

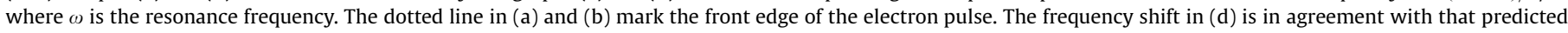
from Fig. 2(a).

a

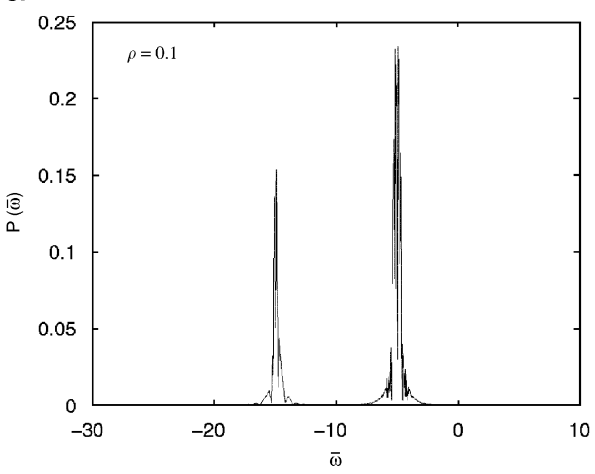

C

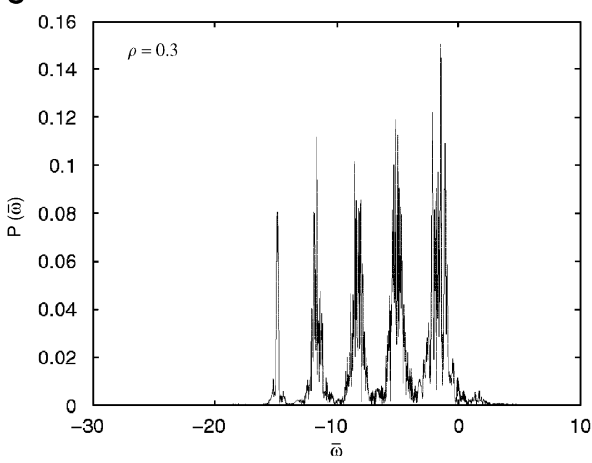

b

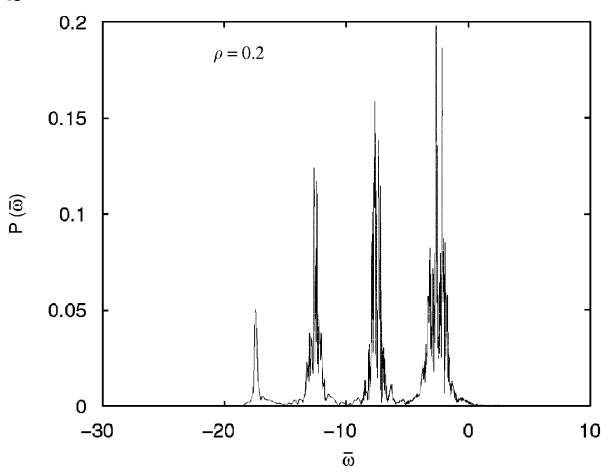

d

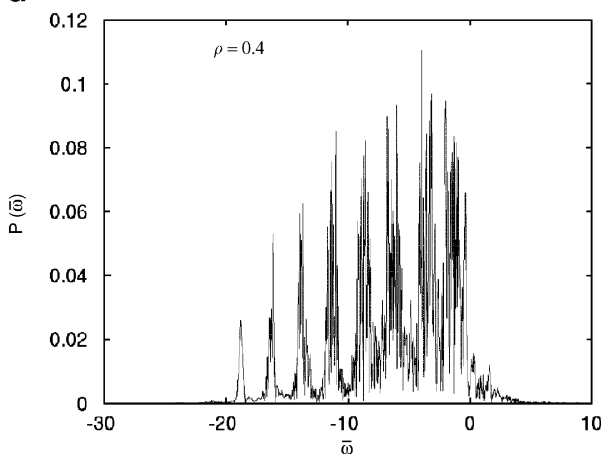

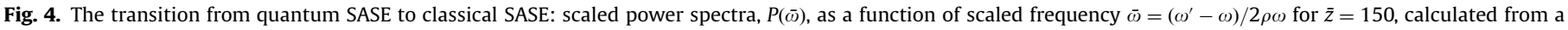
numerical solution of Eqs. (27) and (28) for $\delta=0$ when (a) $\bar{\rho}=0.1$, (b) $\bar{\rho}=0.2$, (c) $\bar{\rho}=0.3$, and (d) $\bar{\rho}=0.4$. 


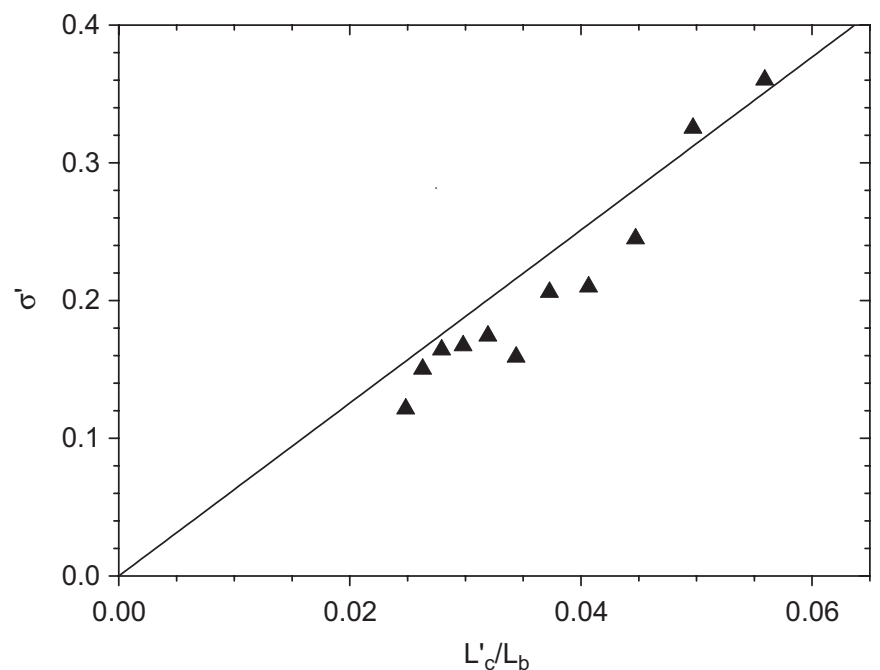

Fig. 5. Line width of the single spike in the quantum regime, $\sigma^{\prime}=\left(L_{c}^{\prime} / c\right) \sigma_{\omega}$, vs. the inverse of the electron bunch length, $L_{\mathrm{c}}^{\prime} / L_{\mathrm{b}}$ (triangles). The straight line is $\sigma^{\prime}=2 \pi\left(L_{\mathrm{c}}^{\prime} / L_{\mathrm{b}}\right)$.

This means that a QFEL operating in the Ångstrom region with the electron beam duration $\tau=1 \mathrm{ps}$ could generate radiation with a line width of $10^{-7}$, much smaller than the envelope line width $2 \rho$ of the classical SASE spectrum (typically of the order of $10^{-3}$ ). Hence, the QFEL can be a very promising X-ray source generating quasi-monochromatic radiation (although at a lower power than in a classical SASE-FEL) and a formidable tool for ultra-high resolution process studies.

We have done a preliminary numerical test of Eq. (30), measuring the width $\sigma^{\prime}=\left(L_{\mathrm{c}}^{\prime} / c\right) \sigma_{\omega}$ of the single spike in the SASE spectrum for different electron bunch lengths $L_{\mathrm{b}} / L_{\mathrm{c}}^{\prime}$, where $L_{\mathrm{c}}^{\prime}=$ $L_{\mathrm{c}} / \sqrt{\bar{\rho}}$ is the quantum cooperation length. The simulations have been done for $\bar{\rho}=0.2$ and the SASE spectrum is at $\bar{z}=20$ (i.e. after nine quantum gain lengths $L_{\mathrm{g}}^{\prime}=L_{\mathrm{g}} / \sqrt{\bar{\rho}}$ ). Fig. 5 shows $\sigma^{\prime}$ as a function of $L_{\mathrm{c}}^{\prime} / L_{\mathrm{b}}$ (triangles) and the prediction of Eq. (30), which in terms of dimensionless variables reads $\sigma^{\prime}=2 \pi\left(L_{\mathrm{c}}^{\prime} / L_{\mathrm{b}}\right)$ (straight line in Fig. 5). The numerical points of $\sigma^{\prime}$ lay around Eq. (30), proving that the width of the spike is Fourier limited by the electron pulse duration. Hence, the coherence length in the quantum regime is of the order of the electron beam length. A more complete numerical study of the quantum line width in SASE will be presented elsewhere.

\section{Conclusions}

In conclusion, we have reviewed some aspects of the quantum regime of the FEL (QFEL). Firstly, from a quantum $N$-particle FEL theory we have obtained the cubic characteristic equation, showing the shift and the narrowing of the FEL resonance in the quantum regime $\bar{\rho}<1$. The same results have been obtained also using the second quantization formalism, from which it has been shown that photons and electrons can be entangled [15]. In the second part, we have presented the principles of the quantum SASE regime, whose dynamical properties appear very different from the usual classical SASE. In contrast to what happens in the classical regime, in the quantum limit $\bar{\rho}<1$ a "quantum purification" of the temporal and spectral structure occurs: the spectrum becomes a series of discrete narrow lines, separated in momentum space by $\hbar k$ and with a width $4 \bar{\rho}^{3 / 2}(\hbar k)$. The continuous and broad spectrum observed in the classical SASE is recovered when $\bar{\rho}$ increases such that the width of each discrete line exceeds the separation between the lines, so that they overlap. A simple interpretation of the observed spectral line width, Fourier limited by the electron beam length, is given. The possibility of experimental operation in the quantum regime using a laser wiggler has been investigated in Refs. [9-11]. In this prospective, a complete three-dimensional quantum model for an FEL with a laser wiggler, based on the Wigner function formalism, has been developed [20] and is currently under investigation [21].

\section{References}

[1] R. Bonifacio, C. Pellegrini, L. Narducci, Opt. Commun. 50 (1984) 373.

[2] The LCLS design study group: LCLS design study report, SLAC-R521, Stanford, 1998 and 〈http://www-ssrl.slac.stanford.edu/lcls/CDR〉.

[3] R. Brinkmann et al., TESLA XFEL: first stage of the LCLS design study report, SLAC-R521, Stanford, 1998 and 〈http://www-ssrl.slac.stanford.edu/lcls/CDR〉.

[4] T. Shintake, Status of the SCSS test accelerator and XFEL project in Japan, EPAC'06, Edinburgh, 2006 and 〈http://www-xfel.spring8.or.jp〉.

[5] R. Bonifacio, L. De Salvo, P. Pierini, N. Piovella, C. Pellegrini, Phys. Rev. Lett. 73 (1994) 70.

[6] R. Bonifacio, M.M. Cola, N. Piovella, G.R.M. Robb, Europhys. Lett. 69 (2005) 55

[7] R. Bonifacio, N. Piovella, G.R.M. Robb, Nucl. Instr. and Meth. A 543 (2005) 645.

[8] R. Bonifacio, N. Piovella, G.R.M. Robb, A. Schiavi, Phys. Rev. ST Accel. Beams 9 (2006) 090701.

[9] R. Bonifacio, N. Piovella, M.M. Cola, L. Volpe, Nucl. Instr. and Meth. A 577 (2007) 745

[10] R. Bonifacio, Nucl. Instr. and Meth. A 546 (2005) 634.

[11] R. Bonifacio, M. Ferrario, G.R.M. Robb, N. Piovella, A. Schiavi, L. Serafini, in: Proceedings of the 27th International Free Electron Laser Conference, vol. 71, 2005.

[12] R. Bonifacio, F. Casagrande, Opt. Commun. 50 (1984) 251.

[13] R. Bonifacio, F. Casagrande, Nucl. Instr. and Meth. A 237 (1985) 168.

[14] G. Preparata, Phys. Rev. A 38 (1988) 233.

[15] N. Piovella, M. Cola, R. Bonifacio, Phys. Rev. A 67 (2003) 013817.

[16] R. Bonifacio, N. Piovella, G.R.M. Robb, M.M. Cola, Opt. Commun. 252 (2005) 381.

[17] G.T. Moore, M.O. Scully, Phys. Rev. A 21 (1980) 2000.

[18] R. Bonifacio, B.W.J. McNeil, P. Pierini, Phys. Rev. A 40 (1989) 4467.

[19] N. Piovella, M. Gatelli, R. Bonifacio, Opt. Commun. 194 (2001) 167.

[20] M.M. Cola, L. Volpe, N. Piovella, A. Schiavi, R. Bonifacio, Nucl. Instr. and Meth. A (2008), doi:10.1016/j.nima.2008.04.046.

[21] A. Schiavi, M.M. Cola, L. Volpe, N. Piovella, R. Bonifacio, Nucl. Instr. and Meth A (2008), doi:10.1016/j.nima.2008.04.064. 